\title{
Comparison of cloud computing providers for development of big data and internet of things application
}

\author{
Muhammad Fajrul Falah ${ }^{1}$, Yohanes Yohanie Fridelin Panduman², Sritrusta Sukaridhoto ${ }^{3}$, \\ Arther Wilem Cornelius Tirie ${ }^{4}$, M. Cahyo Kriswantoro ${ }^{5}$, Bayu Dwiyan Satria ${ }^{6}$, Saifudin Usman $^{7}$ \\ ${ }^{1,2}$ Electrical Engineering Department, Politeknik Elektronika Negeri Surabaya, Indonesia \\ ${ }^{3-7}$ Informatic and Computer Department, Politeknik Elektronika Negeri Surabaya, Indonesia
}

\begin{tabular}{l} 
Article Info \\
\hline Article history: \\
Received Mar 20, 2020 \\
Revised Mar 4, 2021 \\
Accepted Mar 30, 2021 \\
\hline Keywords: \\
Big data \\
Cloud computing \\
Cloud computing comparison \\
Cloud computing providers \\
Internet of things
\end{tabular}

\begin{abstract}
The improved technology of big data and the internet of things (IoT) increases the number of developments in the application of smart city and Industry 4.0. Thus, the need for high-performance cloud computing is increasing. However, the increase in cloud computing service providers causes difficulties in determining the chosen service provider. Therefore, the purpose of this study is to make comparisons to determine the criteria for selecting cloud computing services following the system architecture and services needed to develop IoT and big data applications. We have analyzed several parameters such as technology specifications, model services, data center location, big data service, internet of things, microservices architecture, cloud computing management, and machine learning. We use these parameters to compare several cloud computing service providers. The results present that the parameters able to use as a reference for choosing cloud computing for the implementation of IoT and big data technology.
\end{abstract}

This is an open access article under the CC BY-SA license.

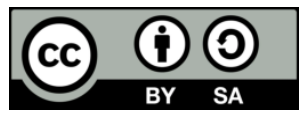

\section{Corresponding Author:}

Sritrusta Sukaridhoto

Informatics and Computer Department

Politeknik Elektronika Negeri Surabaya

Surabaya, East Java, Indonesia

Email: dhoto@pens.ac.id

\section{INTRODUCTION}

With current technological advancements, such as the internet of things (IoT), cyber-physical systems (CPS), and blockchain that are applied to industry era 4.0 [1], in the field of internet of things, various studies are showing that the IoT market will grow rapidly. It is estimated that the development of the number of IoT devices used in 2025 will reach 64 billion [2]. In other research by Cisco appraised that the quantity of embedded systems device associated with the Internet in 2020 will arrive at 50 billion [3]. The ability of IoT systems to integrate with various fields is the reason why the number of IoT technology implementations is increasing rapidly [4], [5].

Because of that, the need for cloud computing is increasing [6]. Cloud computing is a computing model that makes it possible to access information or data through the internet network comfortably and ondemand to a collection of computing resources that can be configured together [7]. In general, the types of cloud computing services consist of infrastructure as a service (IaaS), platform as a service (PaaS) and software as a service (SaaS). Several studies that estimate the development of the size of the global cloud computing market, such as research published by TC 1228 showing that from USD 272.0 billion in 2018 it is estimated to be USD 623.3 billion in 2023 [8], [9]. With this large market, many cloud computing providers provide services that facilitate development, both in functional and non-functional needs. 
There are research on the comparison of cloud computing, such as research conducted by Maurizio Naldi and the team shows a comparison method of cloud computing providers based on the price of the services provided [10]. Meanwhile, research published by Filiopoulou [11] presents an approach that focuses on calculating service efficiencies such as computation optimized instances, memory optimized instances, and storage optimized instances [11]. Other publications conducted by Junjie Peng use the functional requirements of the cloud computing services provided [12]. But there are no other comparison parameters such as server location, this is related to the rules of data center usage that must exist in the country, such as the rules in Indonesia. Therefore, the need a comparison parameter is needed by the developer or user in choosing the provider and optimal cloud computing services, according to the needs and existing rules for build an IoT application that is integrated with big data services.

This journal is divided into several sections. Section 1 describes the need for cloud computing for IoT systems which is the background problem of this research. In section 2, we show a comparison method for cloud computing service providers that have been worked on by previous researchers. In section 3 , we describe the comparison method of cloud computing service providers proposed in this study. The results of the comparison process are described and discussed in section 4. Finally, in section 5 we explain the conclusions of this research and the future works that we plan to do.

\section{RELATED WORKS}

There are several studies about the comparison of cloud computing providers that proposed some parameters or criteria used to discuss the performance of cloud service for cloud computing providers selection. The most commonly shown approach is the price parameter [10], [13], [14], research by Maurizio Naldi and Groups [10] about reviewed and analyzed the estimating plans of a determination cloud service utilizing the unit cost with two strategy approaches, the first technique utilized a pointwise examination for each estimation of capacity volume, by distinguishing the least expensive cloud supplier for every limit section. The second strategy utilizing a two-part tariff approximation and Pareto-predominance criteria for all the evaluating plans considered and utilize that guess to arrange them. As research conducted by Li et al. [15] shows the relationship between the cost of cloud computing with the performance obtained using two metrics, namely cluster computing, and storage services. This method first uses monetary costs to complete benchmarking tasks to capture the effectiveness of virtual instance costs. Then, use the costs of each storage operation to compare the cost-effectiveness of different storage services.

Other researchers, Brebner and Liu [16] showed the parameters that cloud computing services should have in more detail than previous research. The parameters used in the study are Server performance variability, local area network (LAN) latency and bandwidth, and WAN latency and bandwidth. Research conducted by Pierleoni et al. [17] and the team had compared 3 popular cloud computing providers such as Amazon Web Services, Google Cloud Platform and Microsoft Azure regarding IoT services provided by each provider. Therefore, in this research, we conduct analysis and compilation using several parameters that able to affect the development of IoT and Big Data systems, especially in cloud computing services provided by several providers.

\section{RESEARCH METHOD}

In the current IoT technology development, many cloud computing service providers appear both on an international scale such as Amazon Web services (AWS) and Google cloud platform (GCP), as well as local Indonesian cloud computing service providers such as CloudKilat. Each cloud computing service provider has different technology, features, and data center locations. Therefore, in this study, we aim to compare the features provided by each cloud computing service provider to be used as a cloud computing medium in developing an IoT system that is integrated with big data services to support the needs of computing services such as smart city, or industry 4.0. Therefore, we propose several parameters that able to used for cloud computing services comparison which consist of:

\subsection{Technology specifications}

Each cloud computing provider uses a physical device engine that functions as a data center for the storage and distribution of the server [18]. Typically, cloud computing providers offer different node flavors to optimize computing performance, input/output (I/O), or amount of memory. Therefore, in this study, we consider the server technology specifications used by cloud computing providers.

\subsection{Model services}

The use of pay-per-usage methods in cloud computing services has led to the emergence of several types of service types provided by cloud computing providers [19]. The modeling used includes 
infrastructure as a service (IaaS), software as a service (SaaS), and platform as a service (PaaS). So, in this study, we use the service model as a comparison parameter.

\subsection{Data center location}

Each cloud computing service provider provides data center locations in various places, but not all provide data center locations in every country, the location of the data center affects network latency [20]. There are 2 things to consider when choosing a cloud computing service provider based on the location of the data center. If the system requires very low latency, it is necessary to choose a data center location that is in the same country or closest to the location of the device or user of the IoT system that has been developed. The second reason is about the strict policy of the local government which must choose the location of the data center which is located in the same country as the device location device or IoT system user that has been developed.

\subsection{Big data service}

IoT system is a system that retrieves device data continuously at a certain time and produces a lot of data with various data content. Thus, the IoT system requires big data services to accommodate this data, this is because of the ability of big data services to store large amounts of data, vary and able to analyze data more efficiently [21]. Currently, big data services are developing into a more complex architecture to be used in various requirements, such as real-time analysis processes that are often used by IoT system developers. Big data frameworks that are often used by developers such as Apache Spark and Hadoop.

\subsection{Internet of things}

IoT platforms are currently developing in the market [22], causing several cloud computing services to provide special services for IoT systems. Thus, in this research, we use the internet of things parameters to compare services related to IoT technology such as device management, connectivity management for message management, interfaces for data visualization to services for analytical IoT data obtained.

\subsection{Microservices architecture}

The microservice system in cloud computing services functions as a computational service architecture that divides and groups the services used by users in the form of interconnected microservices. This architecture aims to simplify the complex large-scale system development process and improve the performance of the system. This is because the IoT system development process is dynamic and requires real-time data processing.

\subsection{Cloud computing management}

In the development of the IoT application system, it allows for many situations where the development hires one or more server computers, both in the form of containers or virtual machines. So that cloud computing services generally provide at least a management-related service that allows users to create, monitor, and control cloud computing sessions [23]. Therefore, the parameters about the services used by cloud computing providers in helping users to server management need to be considered. Besides, the existence of the microservice concept applied to an IoT application caused a delay in the role of cloud computing management services. Beside cloud computing, IoT also depend on edge computing. The benefits of edge computing mean that application can move their infrastructure from centralized location to the edge and run application closer to the end user.

\subsection{Machine learning}

In developing an IoT application that is integrated with big data, the implementation of machine learning services is increasing. Therefore, we use machine learning service parameters in this study to compare services used by cloud computing providers, namely how cloud computing providers provide machine learning services as analytic services both in real-time or offline (batch) form. This includes the types of algorithms that can be used such as unsupervised learning, supervised learning and reinforced learning [24]. In addition to using the comparison method based on the parameters mentioned earlier, in this research, we also present an overview of some popular cloud computing providers. Following is an overview and analysis of the specifications, types of services and features provided by cloud computing providers as follows. 


\section{RESULTS AND DISCUSSION}

In this section, we present the experimental results and analysis of the cloud computing service provider comparison process using the parameters proposed in this study. Cloud service providers that have been compared include Alibaba, AWS, GCP, Microsoft Azure, and CloudKilat.

\subsection{Alibaba cloud}

Alibaba provides a special service for IoT systems, namely Alibaba cloud IoT platform [25]. This service provides communication services between the IoT platform and the device nodes of users with low latency and security services to ensure the security of data communications with various measures to ensure the security of communications carried out by users. The advantage offered by the Alibaba Cloud IoT Platform service is the system's ability to integrate with other Alibaba cloud services or application programming interface (API) systems that have previously been developed by users. Besides, this service provides regulatory features to make it easier for users to develop faster computing service systems, such as in the process of collecting and processing user IoT data. Until this paper was written, IoT and data visualization services servers from Alibaba cloud still in Singapore.

\subsection{Web services}

Amazon web services (AWS) is one of the popular cloud computing service providers, AWS provides several services for IoT system development [26], [27] such as communication, processing and storage services integrated with big data technology. The Big Data service that AWS provides consists of several types of big data services such as Amazon Kinesis, AWS Lamda, Amazon EMR, Amazon QuickSight, Amazon Athena and Amazon Elasticsearch Service. Therefore, users can choose the type of Big Data service according to the needs of the system to be developed. In communication and data flow processing, AWS provides several services for streaming data, where on these services users can build an analysis system on the data stream process (data stream analytics). Thus, users can create real-time data processing and analysis systems such as data prediction systems, application logs or IoT telemetry data that run without waiting for all data to be collected. Data that has been processed can be distributed to data lake services, Big Data or other applications developed by users.

AWS provides storage services with other methods such as AWS S3, which is data storage services distributed across several nodes in cloud computing. In addition to the services for implementing MapReduce, users can take advantage of the elastic map reduce (EMR) service. Amazon EMR is a distributed computing service for processing and storing data using Apache Hadoop, an EMR service enabling systems to distribute and process data across user clusters on AWS. Through the EMR service, users able to use Hadoop tools like Spark and Hive to perform big data analytics. S3 and EMR services can be used as alternative services for users who are unable to develop a Big Data and Hadoop service system in a cluster. However, users must consider bandwidth usage. There is an extract, transformation and load (ETL) service that can make it easier for users to process data such as moving or combining data between data stores.

\subsection{Google cloud platform}

One of the cloud computing service providers that are gaining popularity is Google cloud platform (GCP) [28]. GCP offers cloud computing services that provide integrated data analytics services at scale for greater performance and more efficiency costs. The GCP architecture allows users to develop analytical applications using several popular programming languages such as Python, Java, C \#, Node.js, Ruby or PHP.

Big data services provided by GCP consist of BigQuery, cloud Pub/Sub, data studio, cloud composer, cloud storage, cloud dataproc, cloud dataprep, cloud data fusion, cloud dataflow. The advantages offered by GCP are the speed in analyzing data using ANSI SQL and providing an API to integrate the system with other applications that have been developed by users. The system stability offered by GCP allows the distribution and processing of data to be carried out faster without reducing the accuracy and functionality of the data. Besides, GCP provides Business Intelligence tools in the form of a dashboard. The infrastructure on the GCP service is developed with security and system workload in mind so that users can develop a wider range of data analysis applications.

\subsection{Microsoft Azure}

Microsoft Azure is a cloud computing service developed by Microsoft [29]. Microsoft Azure has several service products that implement Big Data and can be used for IoT systems [30]. Microsoft Azure IoT Suite is a special service provided by Azure as a cloud computing solution for IoT system development. With this service, users able to integrate IoT device data sent to the Azure server with data analytics and machine learning systems. Azure HDInsight service is a data distribution service solution using the Hadoop tool offered by Azure with the advantages offered such as cost-effective and fast processing of massive amounts 
of data. This service is supported by several commonly used data processing tools such as Spark, Hadoop, Kafka, LLAP, R, HiveStorm and others. Besides, the Azure HDInsight service allows users to develop various data processing scenarios such as the extract, transform, and load (ETL) method which able to process data from various types of data sources and store them in structured data storages. The data streaming service on Azure HDInsight allows users to receive data from various types of devices.

Microsoft Azure provides Azure data share services for the process of sharing data between users safely and easily, where users can share new data, invite other users or partners to share data. However, data owners can easily control and supervise other users who access the data. With these advantages, users can collaborate with other parties so that they can enrich the analytical process on the system being developed.

\subsection{CloudKilat}

CloudKilat [31] is a cloud computing service aimed at Indonesian consumers. CloudKilat was launched in September 2013 by PT Infinys System Indonesia. CloudKilat services are divided into two types, CloudKilat and Infinyscloud. CloudKilat is specifically for web developers, individual businesses, startups, and MSMEs, while Infinyscloud (managed services) is aimed at enterprise-class businesses.

Infinyscloud has advantages in terms of fully managed services, flexible billing and in-depth technical consultations so that users get peace of mind with Infinyscloud's cloud infrastructure. Infinyscloud users will be able to enjoy world-class cloud services owned by AWS, Google and Microsoft which will be bundled with services managed by Infinyscloud's expert team so that users get a total solution. With fully managed services, 24-hour customer service support, and data centers located in Indonesia. Besides, the presence of Infinyscloud's local data center guarantees data sovereignty that meets PP No.82 of 2012.

\subsection{Table result}

We have made a comparison using this parameter which is shown in Table 1 which is in the first appendix. Table 1 shows that almost all cloud providers provide high computing resources. GCP has the highest specifications among the five cloud providers, where GCP provides up to 11 TB RAM and is equipped with the latest GPU resource, NVIDIA Tesla T4, which has the highest GPU Counting Ability than any other GPU. In terms of services, all cloud providers provide almost all types of cloud computing services (IaaS, PaaS, and SaaS) except CloudKilat. For cloud management, Azure, AWS, and Alibaba provide specific cloud management products like microservice and dedicated servers. GCP offers products that are simpler than others to serve the needs of cloud services and CloudKilat has Kilat VM 2.0 to provide VPS. Alibaba, AWS, GCP, and Azure have also implemented microservices in their services. Most of them use Kubernetes to regulate the use of infrastructure through the efficient distribution of computing resources in various processes in microservices. In terms of IoT and big data services, Azure and AWS have products that provide more specific functions compared to GCP and Alibaba, from data collection to data analysis. GCP and Alibaba wrap their services in one complete package. To serve machine learning needs, Amazon and GCP provide more case options than Alibaba and Azure. From price comparison, GCP offers lower prices compared to other cloud providers with the same specifications. One important point from cloud providers is the location of data centers. Of all the cloud providers being compared, only Alibaba and CloudKilat have data centers located in Indonesia. This can increase the confidence of cloud users to entrust their data to the cloud provider.

\section{CONCLUSION}

Based on the results and analysis conducted to compare several cloud computing providers that are often used by developers, both global providers, and local Indonesian providers, namely Alibaba, AWS, GCP, Microsoft Azure and CloudKilat using several parameters such as technology specifications, service models, data center locations, big data services, the internet of things, micro service architecture, cloud computing management, and machine learning. The results of cloud computing providers comparison describe that almost all cloud providers provide high computing resources. All cloud providers provide almost all types of cloud computing services (IaaS, PaaS, and SaaS), except CloudKilat. Alibaba, AWS, GCP, and Azure have implemented a microservice architecture use Kubernetes to regulate infrastructure and resource. But overall features and specifications, Azure and AWS have products and features that are more specific in implementing IoT and big data technology when compared to other providers. However, based on server location, to increase user confidence, Alibaba Cloud and CloudKilat have the advantage of being located in Indonesia. Therefore, based on the previous statement, it can be said that the parameters developed in this study can be used as a reference for selecting cloud computing providers by developers in developing IoT and big data applications. In future work, we will compare cloud computing service providers by testing the IoT and big data systems using the services and features outlined in this research. 


\section{APPENDIX}

Table 1. Comparison result

\begin{tabular}{|c|c|c|c|c|c|}
\hline Parameters & Alibaba Cloud & AWS & GCP & Azure & CloudKilat \\
\hline Technology & Intel Xeon Scalable & Intel Xeon Scalable & Intel Xeon Scalable & Intel Xeon Scalable & Intel Xeon \\
\hline \multirow[t]{7}{*}{ Specifications } & Processor (Cascade & Processor (Cascade & Processor (Cascade & Processor (Cascade & Scalable \\
\hline & Lake), Up to $768 \mathrm{~GB}$ & Lake), Up to $768 \mathrm{~GB}$ & Lake), Up to & Lake), Up to 4TB & Processor \\
\hline & Memory, SSD Storage, & Memory, SSD Storage, & 11,776TB Memory, & Memory, & (Cascade \\
\hline & Network Bandwidth: 25 & Network Bandwidth: 25 & SSD Storage, & SSD Storage, & Lake), Up to \\
\hline & Gb/s, NVIDIA Tesla & Gb/s, NVIDIA Tesla & Network & NVIDIA Tesla V100 & $32 \mathrm{~GB}$ \\
\hline & V100 & V100 & Bandwidth: 20 & & Memory, \\
\hline & & & $\begin{array}{l}\text { Gb/s, NVIDIA } \\
\text { Tesla T4 }\end{array}$ & & SSD Storage \\
\hline Services & IaaS, PaaS, SaaS & IaaS, PaaS, SaaS & IaaS, PaaS, SaaS & IaaS, PaaS, SaaS & IaaS, PaaS \\
\hline \multirow{9}{*}{$\begin{array}{l}\text { Internet of } \\
\text { Things }\end{array}$} & IoT Platform, Link Kit & AWS IoT Greengrass, & Cloud IoT Core & Azure IoT Central, & - \\
\hline & SDK & FreeRTOS, AWS IoT & & Azure IoT solution & \\
\hline & & Core, AWS IoT Device & & accelerators, Azure & \\
\hline & & Defender, AWS IoT & & IoT Edge, Azure IoT & \\
\hline & & Device Management, & & Hub, Azure Digital & \\
\hline & & AWS IoT Analytics & & Twins, Azure Time & \\
\hline & & & & Series Insights, & \\
\hline & & & & Azure Sphere, Azure & \\
\hline & & & & Maps & \\
\hline \multirow[t]{12}{*}{ Big Data } & MaxCompute, E- & Amazon Athena, & BigQuery, Cloud & Azure Databricks, & - \\
\hline & MapReduce, & Amazon CloudSearch, & Dataflow, Cloud & Azure Stream & \\
\hline & DataWorks, Realtime & Amazon Elasticsearch & Dataproc, Cloud & Analytics, Azure & \\
\hline & Compute, Elasticsearch, & Service, Amazon EMR, & Pub/Sub, Cloud & Synapse Analytics, & \\
\hline & Data Lake Analytics, & Amazon Kinesis, & Data Fusion, Cloud & HDInsight, Data & \\
\hline & DataV, Quick BI & Amazon Managed & Composer, Data & Factory, Data Lake & \\
\hline & & Streaming for Apache & Catalog, Google & Analytics, Azure & \\
\hline & & Kafka, Amazon Redshift & Data Studio, & Analysis Services, & \\
\hline & & Formation & Google Sheets, & Azure Data Lake & \\
\hline & & & Cloud Dataprep, & Storage, Azure Data & \\
\hline & & & Cloud Data & Explorer, Azure Data & \\
\hline & & & Transfer & Share & \\
\hline Cloud & Elastic Compute & Amazon Elastic & Compute Engine, & Virtual Machines, & Kilat VM \\
\hline \multirow[t]{16}{*}{ Management } & Service, Simple & Compute Cloud (EC2), & App Engine, Cloud & Azure Kubernetes & 2.0, Kilat \\
\hline & Application Server, & Amazon Lightsail, & GPUs, Migrate for & Service (AKS), & Storage, \\
\hline & Elastic GPU Service, & Amazon Elastic & Compute Engine, & Service Fabric, App & Kilat Plesk, \\
\hline & Auto Scaling, Server & Container Service (ECS) & Preemptible VMs, & Service, Container & Kilat \\
\hline & Load Balancer, & Amazon Elastic & Shielded VMs, & Instances, Batch, & Backup, \\
\hline & Container Service for, & Kubernetes Service & Sole-tenant nodes & SQL Server on & Kilat \\
\hline & Kubernetes, Elastic & (EKS), AWS Lambda, & & Virtual Machines, & Hosting, \\
\hline & Container Instance, & VMware Cloud on & & Cloud Services, SAP & Kilat Iron \\
\hline & Container Registry, ECS & AWS, AWS Local & & HANA on Azure & \\
\hline & Bare Metal Instance, & Zones, Amazon EC2 & & Large Instances, & \\
\hline & Super Computing & Spot Instances, Amazon & & Azure Functions, & \\
\hline & Cluster, Web App & EC2 Autoscaling, AWS & & Virtual Machine & \\
\hline & Service, Function & Batch, , Elastic Load & & Scale Sets, Linux & \\
\hline & Compute, Batch & Balancing (ELB) & & Virtual Machines, & \\
\hline & Compute, Dedicated & & & Windows Virtual & \\
\hline & Host & & & Desktop & \\
\hline Machine & Image Search, Machine & Amazon SageMaker, & AI building blocks, & Cognitive Services, & - \\
\hline \multirow[t]{15}{*}{ Learning } & Translation, Machine & Amazon Augmented AI, & AutoML, Vision & Azure Bot Service, & \\
\hline & Learning Platform For & Amazon CodeGuru & AI, Video AI, & Machine Learning, & \\
\hline & AI, Intelligent Speech & (Preview), Amazon & Cloud Natural & Azure Databricks, & \\
\hline & InteractionBeta & Comprehend, Amazon & Language, & Azure Cognitive & \\
\hline & & Lex, & Dialogflow, & Search & \\
\hline & GCP & AWS Deep Learning & AutoML Tables & & \\
\hline & & AMIs, AWS Deep & (beta), AI Platform & & \\
\hline & & Learning Containers, & Deep Learning VM & & \\
\hline & & AWS DeepComposer, & Image, AI Platform & & \\
\hline & & AWS TensorFlow on & Deep Learning & & \\
\hline & & AWS & Containers (beta), & & \\
\hline & & & AI Hub (beta), & & \\
\hline & & & Cloud GPUs, Cloud & & \\
\hline & & & TPU, TensorFlow & & \\
\hline & & & Enterprise & & \\
\hline Microservices & Kubernetes Cluster & AWS ALB, Amazon & Google Kubernetes & Azure Kubernetes & - \\
\hline Architecture & & $\begin{array}{l}\text { ECS, Amazon EKS, } \\
\text { Azure Lambda }\end{array}$ & Engine & Service & \\
\hline
\end{tabular}


Table 1. Comparison result (continue)

\begin{tabular}{|c|c|c|c|c|c|}
\hline Parameters & Alibaba Cloud & AWS & GCP & Azure & CloudKilat \\
\hline $\begin{array}{l}\text { Data Center } \\
\text { Location }\end{array}$ & $\begin{array}{l}\text { Global, Local } \\
\text { (Jakarta) }\end{array}$ & $\begin{array}{l}\text { Global, Local (Jakarta, } \\
\text { est 2021) }\end{array}$ & $\begin{array}{l}\text { Global, Local (Jakarta, } \\
\text { est 2021) }\end{array}$ & Global & Local (Jakarta) \\
\hline Price (USD) & $\$ 1,554.9 /$ month & $\$ 1,701.51 /$ month & $\$ 856.98 /$ month & $\begin{array}{l}\$ 1,332.46 \\
\text { /month }\end{array}$ & $\begin{array}{l}\$ 180.93 \\
\text { /month* }\end{array}$ \\
\hline
\end{tabular}

*Specification: 32 vCPUs, 32 GB RAM and 320GB SSD

\section{ACKNOWLEDGEMENT}

This research was funded from "Applied Leading Research in Higher Education (in Indonesian Penelitian Terapan Unggulan Perguruan Tinggi)" by KEMENRISTEKDIKTI in 2020 with the funding scheme number "130/SP2H/AMD/LT/DRPM/2020".

\section{REFERENCES}

[1] L. D. Xu, E. L. Xu, and L. Li, "Industry 4.0: state of the art and future trends," International Journal of Production Research, vol. 56, no. 8, pp. 2941-2962, Sep. 2018, doi: 10.1080/00207543.2018.1444806.

[2] Newman, Peter, "IoT report: How Internet of Things technology growth is reaching mainstream companies and consumers," Retrieved February 16, 2019.

[3] D. Evans, "The internet of things: How the next evolution of the internet is changing everything," CISCO white paper, vol. 1, pp. 1-11, 2011.

[4] H.-N. Dai, Z. Zheng, and Y. Zhang, "Blockchain for Internet of Things: A Survey," IEEE Internet of Things Journal, vol. 6, no. 5, pp. 8076-8094, 2019, doi: 10.1109/JIOT.2019.2920987.

[5] Y. Y. F. Panduman, A. R. A. Besari, S. Sukaridhoto, R. P. N. Budiarti, R. W. Sudibyo, and F. Nobuo, "Implementation of Integration VaaMSN and SEMAR for Wide Coverage Air Quality Monitoring," TELKOMNIKA Telecommunication Computing Electronics and Control, vol. 16, no. 6, p. 2630, Jan. 2018, doi: 10.12928/telkomnika.v16i6.10152.

[6] D. Dempsey and F. Kelliher, Industry Trends in Cloud Computing: alternative business-to-business revenue models, London, Britania Raya, United Kingdom: Palgrave Macmillan, 2018.

[7] K. Keahey, R. Figueiredo, J. Fortes, T. Freeman, and M. Tsugawa, "Science clouds: Early experiences in cloud computing for scientific applications," Cloud computing and applications 2008, pp. 825-830, 2008.

[8] Cloud Computing Market Predicted to expand \$623.3 billion by 2023: Markets and Markets, Market Research Firm, [Online]. Available: https://www.marketsandmarkets.com/PressReleases/cloud-computing-market.asp. (Accessed: 05-Mar-2020).

[9] G. Zhang and M. N. Ravishankar, "Exploring vendor capabilities in the cloud environment: A case study of Alibaba Cloud Computing," Information \& Management, vol. 56, no. 3, pp. 343-355, 2019.

[10] M. Naldi and L. Mastroeni, "Cloud storage pricing: a comparison of current practices," Proceedings of the 2013 international workshop on Hot topics in cloud services-HotTopiCS 13, 2013, doi: 10.1145/2462307.2462315.

[11] E. Filiopoulou, P. Mitropoulou, N. Lionis, and C. Michalakelis, "On the efficiency of cloud providers: A DEA approach incorporating categorical variables," IEEE Transactions on Cloud Computing, pp. 1-1, 2018, doi: 10.1109/TCC.2018.2850889.

[12] J. Peng, X. Zhang, Z. Lei, B. Zhang, W. Zhang, and Q. Li, "Comparison of Several Cloud Computing Platforms," 2009 Second International Symposium on Information Science and Engineering, 2009, pp. 23-27, doi: 10.1109/ISISE.2009.94.

[13] P. Mitropoulou, E. Filiopoulou, C. Michalakelis, and M. Nikolaidou, "Pricing cloud IaaS services based on a hedonic price index," Computing, vol. 98, no. 11, pp. 1075-1089, 2016, doi: 10.1007/s00607-016-0493-x.

[14] I. Lee, "Developing pricing strategies for cloud service providers in a competitive market," 2018 IEEE/ACM International Conference on Utility and Cloud Computing Companion (UCC Companion), 2018, pp. 387-392, doi: 10.1109/UCC-Companion.2018.8653578.

[15] A. Li, X. Yang, S. Kandula, and M. Zhang, "CloudCmp comparing public cloud providers," Proceedings of the 10th annual conference on Internet measurement-IMC 10, pp. 1-14, 2010, doi: 10.1145/1879141.1879143.

[16] P. Brebner and A. Liu, "Performance and Cost Assessment of Cloud Services," Service-Oriented Computing Lecture Notes in Computer Science, pp. 39-50, 2011, doi: 10.1007/978-3-642-19394-1_5.

[17] P. Pierleoni, R. Concetti, A. Belli, and L. Palma, "Amazon, Google and Microsoft Solutions for IoT: Architectures and a Performance Comparison,” IEEE Access, vol. 8, pp. 5455-5470, 2020, doi: 10.1109/ACCESS.2019.2961511.

[18] M. G. Mayo, J. Duncan, P. P. Candel, J. A. Hoffman, and D. P. Young, "Provisioning server resources in a cloud resource,"U.S. Patent No. 9,021,046. 28 Apr. 2015.

[19] D. Freet, R. Agrawal, S. John, and J. J. Walker, "Cloud forensics challenges from a service model standpoint: IaaS, PaaS and SaaS," Proceedings of the 7th International Conference on Management of computational and collective intElligence in Digital EcoSystems, pp. 148-155, 2015, doi: 10.1145/2857218.2857253.

[20] T. Guo, P. Shenoy, K. K. Ramakrishnan, and V. Gopalakrishnan, "Latency-aware virtual desktops optimization in distributed clouds," Multimedia Systems, vol. 24, no. 1, pp. 73-94, 2017, doi: 10.1007/s00530-017-0536-y. 
[21] C. Yang, Q. Huang, Z. Li, K. Liu, and F. Hu, "Big Data and cloud computing: innovation opportunities and challenges," International Journal of Digital Earth, vol. 10, no. 1, pp. 13-53, Mar. 2016, doi: 10.1080/17538947.2016.1239771.

[22] H. Hejazi, H. Rajab, T. Cinkler, and L. Lengyel, "Survey of platforms for massive IoT," 2018 IEEE International Conference on Future IoT Technologies (Future IoT), 2018, pp. 1-8, doi: 10.1109/FIOT.2018.8325598.

[23] D. D. Spaltro, A. Polvi, and L. Welliver, "Methods and systems for cloud computing management," U.S. Patent No. 9,501,329, 22 Nov. 2016.

[24] U. S. Shanthamallu, A. Spanias, C. Tepedelenlioglu, and M. Stanley, "A brief survey of machine learning methods and their sensor and IoT applications," 2017 8th International Conference on Information, Intelligence, Systems \& Applications (IISA), 2017, pp. 1-8, doi: 10.1109/IISA.2017.8316459.

[25] M. Saraswat and R. C. Tripathi, "Cloud Computing: Comparison and Analysis of Cloud Service Providers-AWs, Microsoft and Google," 2020 9th International Conference System Modeling and Advancement in Research Trends (SMART), 2020, pp. 281-285, doi: 10.1109/SMART50582.2020.9337100.

[26] Amazon Web Services, Inc., Features | Amazon Cloud Directory | Amazon Web Services (AWS), 2020. [online] Available at: https://aws.amazon.com/cloud-directory/features/ (Accessed 14 March. 2020).

[27] Amazon Web Services, Inc., AWS IoT Applications \& Solutions, 2020. [online] Available at: https://aws.amazon.com/iot/ (Accessed 14 March. 2020).

[28] J. Shah and D. Dubaria, "Building Modern Clouds: Using Docker, Kubernetes \& Google Cloud Platform," 2019 IEEE 9th Annual Computing and Communication Workshop and Conference (CCWC), 2019, pp. 0184-0189, doi: 10.1109/CCWC.2019.8666479.

[29] G. Kaur, "Analysis of Virtual Machine Instances and Cost Computation on Microsoft Azure Cloud Service Provider," Journal of Advanced Research in Dynamical and Control Systems, vol. 12, no. 3, pp. 268-278, 2020.

[30] Financesonline, Microsoft Azure Reviews: Pricing \& Software Features 2020 - Financesonline.com, 2020. [online] Available at: https://reviews.financesonline.com/p/microsoft-azure/ (Accessed 14 March. 2020).

[31] Infinys System Indonesia, SSD Cloud Hosting \& Web Hosting - CloudKilat, 2020. [online] Available at: https://www.cloudkilat.com/ (Accessed 19 March. 2020). 